\title{
LA RENOVACIÓN DEL LENGUAJE JURISDICCIONAL EN LA JURISPRUDENCIA DEL TRIBUNAL CONSTITUCIONAL *
}

JAVIER SALAS

Catedrático de Derecho Administrativo Secretario General del Tribunal Constitucional

* Ponencía presentada el día 9 de junio de 1987 en el marco del all Ciclo sobre Administración y Lenguaje», organizado por el "Centro de Estudios y Documentación», dependiente del «Instituto Nacional de Administración Pública», y celebrado en Madrid durante los meses de mayo y junio de 1987. Agradezco muy vivamente al Director de dicho Centro, D. Jesús Moreno, la autorización para publicar la mencionada Ponencia en esta Revista. 


\title{
LA RENOVACIÓN DEL LENGUAJE JURISDICCIONAL EN LA JURISPRUDENCIA DEL TRIBUNAL CONSTITUCIONAL
}

\author{
POR \\ JAVIER SALAS \\ Catedrático de Derecho Administrativo \\ Secretario General del Tribunal Constitucional
}

\section{INTRODUCCIÓN}

Aunque dentro del Ciclo sobre "Administración y Lenguaje», el tema objeto de mi exposición, al referirse al Tribunal Constitucional, merece alguna aclaración preliminar.

El Tribunal Constitucional no es, obviamente, Administración pública, ni está encuadrado dentro de la misma. Es un «órgano constitucional», concepto éste que doy aquí por conocido, y sobre el cual, por consiguiente, no voy a extenderme, sino, simplemente; remitirme a la doctrina elaborada sobre dicha expresión.

Pues bien, en cuanto órgano constitucional, el Tribunal Constitucional, además de ser el «intérprete supremo de la Constitución», es «independiente de los demás órganos constitucionales y está sometido sólo a la Constitución» y a su Ley Orgánica (art. 1.1 de la Ley Orgánica 2/1979, de 3 de octubre; en adelante, LOTC).

Es cierto, sin embargo, que a parte del ejercicio de las funciones especificas y características que le están atribuidas por la Constitución, su Ley Orgánica (LOTC) y otras Leyes Orgánicas posteriores (concretamente, la $3 / 1984$, reguladora de la iniciativa legislativa popular, la $8 / 1984$, por la que se regula el régimen de recursos en caso de objeción de conciencia y la 5/1985, del Régimen Electoral General) y, en concreto, el conocimiento y resolución de los recursos y cuestiones de inconstitucionalidad, el recurso de amparo, los conflictos de competencia entre el Estado y las Comunidades Autónomas y los de éstas entre si, los conflictos entre órganos constitucionales, la impugnación de disposiciones sin fuerza de Ley y resoluciones de las Comunidades Autónomas, asi como la declaración sobre la constitucionalidad de los Tratados internacionales, el Tribunal Constitucional es también un órgano o, mejor, una organización que desarrolla otras funciones que ya no son específicas del mismo y que, desde el punto de vista material, son similares a las de las Administraciones públicas, a las de los órganos de éstas. 
En efecto, el Tribunal Constitucional contrata, a través de los distintos sistemas previstos en la legislación general del Estado, con empresas públicas o privadas, para atender sus necesidades materiales de bienes y servicios internos, tiene a su servicio personal funcionario propio (que integra el Cuerpo de Letrados) o procedente de la Administración civil del Estado y de la Administración de justicia e, incluso, personal en régimen de derecho laboral.

Así, las resoluciones del Secretario General en materia de personal son recurribles en alzada ante el Presidente del Tribunal Constitucional, cuya decisión agota la vía administrativa, decisión que es susceptible de ulterior recurso contencioso-administrativo (art. 99.3 de la LOTC) y, en concreto, ante la Sala de lo Contencioso-administrativo del Tribunal Supremo (art. 58.1 de la Ley Orgánica 6/1985, del Poder Judicial).

Pero no es a estas funciones materialmente administrativas, ni a su reflejo en la correspondiente documentación, a la expresión por escrito, en definitiva, al lenguaje que se utiliza en las mismas, a las que voy a aludir en esta ocasión. Ni tampoco a la que se emplea por órganos no jurisdiccionales del Tribunal Constitucional (a parte de la de la Secretaría General, ya citada, la de la Jefatura del Gabinete del Presidente o la del Servicio de Prensa, dependiente del mismo; en el primer caso, en publicaciones como el folleto de divulgación sobre la actividad y competencias del Tribunal o la reciente "Memoria», que, con ocasión de la conmemoración del sexto aniversario de su entrada en funcionamiento, se editaron para su difusión; en el segundo, a través de notas o declaraciones a los medios de comunicación social, normalmente, relativas a decisiones adoptadas por aquẻl).

Es, concretamente, el lenguaje del que se sirve el Tribunal Constitucional para emanar sus decisiones propiamente jurisdiccionales el objeto de esta Ponencia. Porque, aunque no está, efectivamente - a diferencia de lo que ocurre, por ejemplo, en la República Federal de Alemania: artículos 92 y siguientes de la Ley Fundamental de Bonn-, integrado en el Poder Judicial -como lo prueba no sólo la ubicación del tratamiento constitucional de uno y otro en Títulos diferentes, el VI y el IX, respectivamente, de la Constitución, sino por el hecho, de que, tanto según ésta, como, sobre todo, según la LOTC, ef Tribunal Constitucional controla la actividad de los demás Poderes del Estado, el legislativo, el ejecutivo y el judicial-, ejerce típicamente una función judicial o jurisdiccional, como lo demuestra no sólo el procedimiento que se sigue ante el mismo para resolver los asuntos de los que es competente, sino también la forma que adoptan sus resoluciones en ese ámbito, que no son otras que aquellas mediante las cuales expresan su voluntad los órganos judiciales y, en concreto, los integrados en el Poder Judicial, en la jurisdicción llamada ordinaria.

Voy, pues, a ceñirme al lenguaje utilizado por el Tribunal Constitucional a través de sus decisiones típicas, propias o caracteristicas del mismo como "órgano constitucional», decisiones que revisten la forma de 
providencias, autos y sentencias. Será a estos dos tipos de resoluciones a las que habré de referirme, concretamente, dentro de un momento.

\section{LOS ANTECEDENTES}

Permítaseme, no obstante, antes de entrar, directamente, en el tema enunciado, hacer algunas alusiones al lenguaje utilizado tanto por los órganos del Poder Judicial y, más en concreto, por el Tribunal Supremo, como por el antecedente histórico del actual Tribunal Constitucional, el Tribunal de Garantias Constitucionales de la II República española y por otros Tribunales Constitucionales extranjeros o equivalentes (la Corte Suprema de los Estados Unidos de Norteamérica, el Tribunal Constitucional Austríaco, el Tribunal Constitucional Federal Alemán, la Corte Constitucional italiana, el Consejo Constitucional francés y el Tribunal Constitucional portugués).

Como la perspectiva que aquí me interesa destacar es, fundamentalmente, la comparativa, voy a fijarme, en primer término, en el lenguaje empleado por el Tribunal Supremo en las decisiones que del mismo se publican.

Pues bien, aun a riesgo de incurrir en un cierto simplísimo, por la, quizá excesiva, generalización que voy a hacer, puedo decir que la expresión por escrito, el lenguaje utilizado por el Tribunal Supremo en sus decisiones ha pecado -insisto, en general, con todas las excepciones que se quieran, que las ha habido, ciertamente-, de ser algo farragoso, de escasa claridad, a veces, incluso, de difícil comprensión, no ya por parte de profanos, sino, incluso, de juristas (Abogados y Profesores de Derecho), de, en definitiva, y por resumir, de poco cuidado en la utilización, desde el punto de vista sintáctico, del "estilo», digamos, literario, del castellano.

¿Cuáles han podido ser las causas de ese fenómeno, de cuya existencia se habrán, sin duda, percatado quienes hayan manejado las distintas colecciones de jurisprudencia?

A mi juicio, son varias. En primer lugar, las formulaciones exigidas por la Ley de Enjuiciamiento Civil (de 1881) y de Enjuiciamiento Criminal (de 1882), en cuyos artículos 371 y $372.2 .^{\circ}$ y 141 y 142 , respectivamente, se habla de "resultandos" y "considerandos" como fórmula para comenzar la exposición de las pretensiones de las partes y de los hechos en que las funden y los fundamentos doctrinales y legales que se estimen procedentes para el fallo que haya de dictarse.

En segundo término, la escasez de medios (personales y materiales) y de tiempo para redactar con la mayor corrección estilística las decisiones. Ante el verdadero «alud» de asuntos que llegan, no sólo a los Juzgados y Tribunales inferiores, sino también al Tribunal Supremo $-\mathrm{y}$ me remito a 
los datos suministrados en las "Memorias" que tan cuidadosamente elabora, con periodicidad anual, el Consejo General del Poder Judicial-, los miembros de las distintas Salas del mismo y, en concreto, quienes redactan las diferentes resoluciones (los Ponentes), han optado por primar el factor celeridad, en vez de hacerlo por el valor claridad y elegancia estilística, en definitiva, y así podría formularse, por la rapidez en resolver, dentro de todas las limitaciones existentes, los asuntos, en vez de por el cuidado en el estllo literario, en la elegancia lingüistica y la claridad expositiva.

Por último, la escasa preocupación por la utilización del castellano en toda su riqueza léxica y armazón sintáctica.

Por lo que respecta al Tribunal de Garantias Constitucionales, puedo decir que sus Sentencias están, por lo general, bien redactadas, no son extensas, pero tampoco demasiado breves; tienen una estructura diferente a la de las Sentencias de los Tribunales ordinarios, sin sujetarse, normalmente, al «pie forzado» del gerundio («resultandos» y «considerandos»). Aunque no siempre —debido, quizá, a la impronta personal o procedencia profesional del Ponente-, se utiliza en la inmensa mayoría de aquéllas la fórmula de «antecedentes» $y$ «fundamentos legales».

\section{EL LENGUAJE UTILIZADO POR OTROS TRIBUNALES CONSTITUCIONALES}

En lo que concierne al lenguaje utilizado por otros Tribunales Constitucionales, he de señalar el contraste notorio existente entre el estilo del Conseil Constitutionnel y el de los demás Tribunales a los que antes he hecho referencia.

En efecto, el primero, salvo contadas excepciones - pueden citarse, en este sentido, entre otras, las decisiones «Securité et liberté» de 19-20 de enero de 1981, «Nationalisations», de 16 de enero y de 11 de febrero de 1982, «Perquisitions fiscales», de 29 de diciembre de 1983, "Libertés universitaires", de 20 de enero de 1984, «Entreprises de Presse», de 10-11 de octubre de 1984, «Mesures d'ordre économique et social», de 25-26 de junio de 1986, y “Liberté de communication», de 18 de septiembre de 1986-, es tributario, sin duda, de la técnica empleada por el Conseil dÉtat del mismo país. Son, normalmente, brevísimos, a veces, incluso, apodícticos, los fundamentos que sustentan el fallo. Son los Profesores de Universidad, los famosos arrêtistes, los que han desarrollado los argumentos, los que han "creado" la "doctrina", en un caso, del Derecho Administrativo - Hauriou, Duguit, Jèze, etc.-, en el otro, la del Derecho Constitucional «jurisprudencial»-_especialmente, Louis Favoreu y Loïc Philip-. 
Los demás Tribunales, comenzando por la Corte Suprema de los Estados Unidos de Norteamérica, se caracterizan por ofrecer un amplio desarrollo de sus argumentaciones, caracterizadas por la flexibilidad sintáctica, la mayor o menor belleza literaria $y$, en ocasiones, hasta el fino humor, con citas, con cierta frecuencia, de autores, especialmente, en las Sentencias del Tribunal Constitucional Federal Alemán y del Tribunal Constitucional portugués. En esta línea, muy próxima, a mi juicio, a la seguida por la Corte Costituzionale, se halla, precisamente, el Tribunal Constitucional español.

\section{EL LENGUAJE UTILIZADO POR EL TRIBUNAL CONSTITUCIONAL ESPAÑOL}

A este respecto, podemos destacar cómo el Tribunal Constitucional español ha cuidado desde el principio el estilo literario de sus resoluciones, no sólo de las Sentencias, sino también de los Autos dictados por el mismo. $Y$ es que éste ha hecho un auténtico esfuerzo por depurar la forma de expresión de sus decisiones (como ha puesto tempranamente de relieve el Prof. Lorenzo Martín-Retortillo), rompiendo, decididamente, con el "gerundio", con el pie forzado de los "resultandos» y «considerandos», apelando a la técnica, que permite una mayor libertad expresiva, de los «Antecedentes" y de los «Fundamentos jurídicos». Lo cual, quizá, no deja de tener sus inconvenientes, como puede ser la desviación del «hilo conductor» que lleva directamente a fundamentar el fallo o la introducción de obiter dicta. Pero tales inconvenientes, posibles, desde luego, teóricamente, pero escasos, en mi opinión, en la práctica habida hasta el presente, están muy por debajo de sus innegables ventajas. De ahi que no comparta la crítica, excesivamente dura, a mi juicio, y creo que, sinceramente, equivocada, del Prof. Tomás-Ramón Fernández a la novedad expresiva del Tribunal Constitucional, y, menos aún, su añoranza por el viejo estilo de los Tribunales ordinarios como alternativa de aquélla.

En este sentido, podriamos considerar como características de la expresión del Tribunal Constitucional en el ejercicio de su función jurisdiccional la claridad, el rigor expositivo y hasta el afán didáctico o pedagógico, en cuanto que sus decisiones parecen dirigirse no sólo a las partes del proceso de que se trate, sino también a los órganos de la jurisdicción ordinaria, a los jurístas e, incluso, a los simples ciudadanos (en esta línea puede verse, por ejemplo, por lo que atañe a la finalidad de la motivación de las resoluciones judiciales, la reciente Sentencia de la Sala $1 .^{a} 55 / 1987$, de 13 de mayo). Afán didáctico, digo, que aparece muy claramente en los Autos de inadmisión de los recursos de amparo, sobre todo, en los de los primeros años de funcionamiento del Tribunal Constitucional, a través del empleo, relativamente frecuente, de los argumentos a fortiori o a mayor 
abundamiento, para reforzar el fallo de inadmisión, como si el Tribunal temiera que el solicitante de amparo, que ha visto así rechazado su recurso, fuera a tacharle de haber sido demasiado expeditivo al basar su negativa a admitirlo a trámite en una sola causa, por muy clara que fuese (por ejemplo, la extemporaneidad o la falta insubsanable de un requisito procesal $o$, incluso, de fondo, como la carencia manifiesta de contenido constitucional de la demanda).

Quizá porque ha transcurrido ya un periodo lo suficientemente amplio como para que su doctrina haya sido conocida y asimilada por los Abogados que asisten a los recurrentes, lo cierto es que, desde hace ya unos cuantos meses, se observa una mayor contención del Tribunal a la hora de exponer la fundamentación de las inadmisiones de los recursos de amparo. $Y$ es que no en vano han aparecido, en la fecha en que esto decimos, quince extensos volúmenes de Jurisprudencia Constitucional, lo que ha llevado, por lo demás, al Tribunal, a partir del primer volumen correspondiente a 1986 (el XIV), a seleccionar los Autos que se publican íntegramente en dicha colección. De ahi la mayor frecuencia con que los Autos se limitan a exponer la existencia de un único motivo de inadmisión para basar el fallo de los mismos, aunque se hayan puesto de relieve varias causas en la correspondiente providencia del trámite previsto en el art. 50 de la LOTC.

¿A qué se debe ese «estilo» adoptado por el Tribunal Constitucional? En mi opinión, fundamentalmente, a que se trata de un Tribunal compuesto hasta ahora mayoritariamente por Profesores de Universidad - $y$, en concreto, por Catedráticos de disciplinas jurídicas-, acostumbrados como están a exponer verbalmente y por escrito sus ideas con una ordenación sistemática, una claridad y un rigor expositivos propios del quehacer académico. Un Tribunal, por cierto, con una proporción de Profesores significativamente más elevada que en otros Tribunales Constitucionales, como los antes citados. Profesores que, por añadidura, también abundan entre los Letrados reclutados al servicio de aquél.

No voy a entrar en detalles, que no son del caso a los efectos que persigo en esta exposición, pero quiero notar que, aun dentro de las características estilísticas propias de cada Ponente, en el Tribunal Constitucional español ha habido desde el principio y subsiste, tras la reciente renovación de seis de sus miembros, una cierta diferencia entre las resoluciones de las que son Ponentes quienes proceden de la Academia y las de los que son «autores» quienes provienen de la judicatura ordinaria (de la carrera judicial) o de la Abogacía.

Dicha mayoria ha influido también, a mi modo de ver, en el lenguaje utilizado en sus decisiones como Ponentes de los Magistrados no procedentes de la carrera universitaria. 


\section{CONSIDERACIONES FINALES}

Por lo que respecta al lenguaje empleado por el Tribunal Constitucional español en sus resoluciones jurisdiccionales podría llegarse, a mi juicio, a las siguientes conclusiones:

Primera: La ruptura con el estilo utilizado por los Tribunales ordinarios y, en concreto, por el Tribunal Supremo.

Segunda: La aproximación creciente de las decisiones de éste al lenguaje del Tribunal Constitucional $-\mathrm{y}$ no me refiero, claro es, al apoyo en su jurisprudencia para resolver los asuntos de los que conocen, que éste es otro tema ajeno al presente, en virtud de lo dispuesto tanto en la Constitución (art. 164.1) como en la LOTC (artículos 38.1 y 87.1 ) y en la reciente Ley Orgánica del Poder Judicial (art. 5.1)—, lo cual puede observarse desde hace ya algún tiempo, al menos, en las Sentencias emanadas por determinados Ponentes de las Salas del Tribunal Supremo (y, seguramente, de los demás órganos inferiores a éste).

Tercera: La aparición de la citada Ley Orgánica del Poder Judicial en julio de 1985 ha venido a reforzar, extendiéndola a los Juzgados y Tribunales de la jurisdicción ordinaria, la línea iniciada por el Tribunal Constitucional (art. 248; precepto éste que, por cierto, habla de "hechos" y «razonamientos jurídicos" y de "antecedentes de hecho" y ufundamentos de derecho", respectivamente, en relación con Autos y Sentencias, sin que adivinemos la razón de tal diferencia «formal» en uno y otro caso, por más que sea, por supuesto, trivial y no relevante).

Cuarta: La coincidencia, en líneas generales, del lenguaje utilizado por el Tribunal Constitucional español con el de otros Tribunales homólogos, singularmente, con el de la Corte italiana y, algo menos, con el de los Tribunales alemán y austriaco y con el de la Corte Suprema norteamericana.

Quinta: La diferencia notable del estilo del Tribunal español con el del Conseil Constituionnel, tan parco en palabras, como se ha dicho, salvo en decisiones muy contadas.

Sexta: La posibilidad de emisión de votos particulares o disidentes respecto de la opinión de la mayoría por parte de los Magistrados del Tribunal Constitucional español, permite que aquélla y, en concreto, el Ponente, tenga, normalmente, menos problemas a la hora de la redacción de la sentencia (o del Auto, en su caso) que si no existiera tal posibilidad 
(inexistente, como se sabe, en la Corte Costituzionale y en el Conseil Constitutionnel, se da, también, en la Corte americana y en los Tribunales alemán, austríaco y portugués), posibilidad extendida en nuestro sistema por la Ley Orgánica del Poder Judicial (art. 260) a los Tribunales ordinarios, a cuyos miembros se permitía antes pronunciar, únicamente, «votos reservados", que no se hacian públicos y de los que conocia solamente el Tribunal Supremo si se interponía contra la correspondiente resolución del Tribunal inferior, y era admitido por aquél, el pertinente recurso de casación (art. 368 de la Ley de Enjuiciamiento Civil).

Séptima: La influencia de la previsión de la LOTC (art. 90.2) de los votos particulares y de su práctica por el Tribunal Constitucional en los Tribunales de la jurisdicción ordinaria, práctica que, sin ser tan frecuente como en la Corte Suprema de los Estados Unidos, no es, ni mucho menos, inusual en nuestro Tribunal. Como es obvio, es en los votos particulares -sean individuales o suscritos por varios jueces constitucionales- donde se nota mayormente el estilo personal de sus redactores, que en este supuesto y, salvo que sean firmados por un colectivo de Magistrados (que no es lo mismo que la adhesión de otro u otros jueces al voto particular emitido por uno solo), reflejan la impronta estilistica particular de cada uno de ellos, más que en las decisiones elaboradas por un Ponente cuya tesis sea compartida por todo el Tribunal Constitucional (o por la mayoria de sus componentes), ya que, como es lógico, en este caso el Ponente acepta, normalmente, la incorporación de sugerencias verbales o de textos propuestos por otros colegas a su proyecto de resolución. 